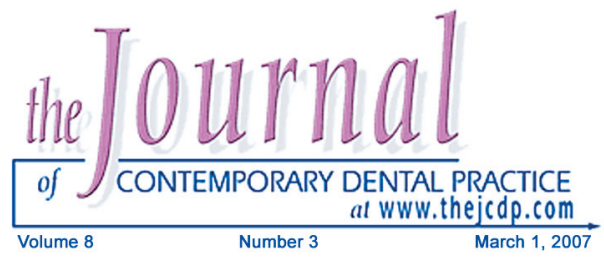

\title{
Hematoma Following Periodontal Surgery with a Torus Reduction: A Case Report
}

\author{
Katherine Mantzikos, DMD, MS; Stuart L. Segelnick, DDS, MS;
}

Robert Schoor, DDS

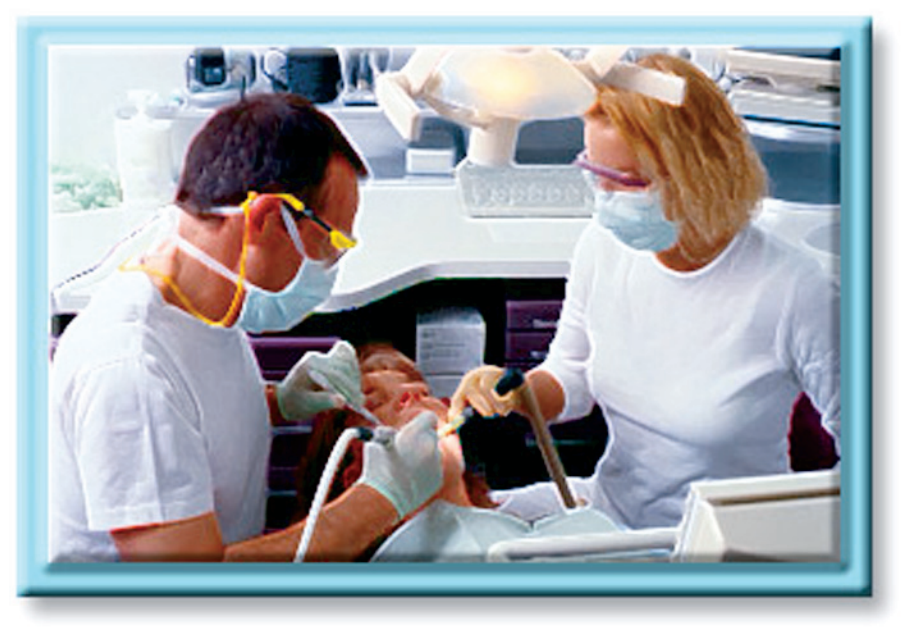

Abstract

Background: There are no published case reports that hematomas occur on the floor of the mouth as a result of periodontal surgery.

Report: These three case reports document post surgical hematoma formation on the floor of the mouth that pose problems with the diagnosis, prognosis, and patient management. All cases involved periodontal surgery where extensive reduction of tori and bony exostoses were required. Two cases resulted in hematomas in the floor of the mouth. However, a change in the post surgical management for a third case demonstrated possible prevention of hematoma formation.

Summary: Hematomas in the floor of the mouth can be a resolving post surgical phenomenon or a serious vascular insult to this region of the oral cavity. This report clarifies the diagnosis, prognosis, and the best management protocol through the presentation of three cases.

Keywords: Hematoma, tori, periodontal surgery, floor of the mouth

Citation: Mantzikos K, Segelnick SL, Schoor R. Hematoma Following Periodontal Surgery with a Torus Reduction: A Case Report. J Contemp Dent Pract 2007 March;(8)3:072-080

(c) Seer Publishing 


\section{Introduction}

The gold standard for bone graft material used in a surgical procedure for bone regeneration is autogenous bone. ${ }^{1}$ Mandibular tori and or thick boney lingual exostoses provide substantial sources of autogenous cortical bone, bone containing quantities of bone morphogenic protein, which is a potent inductive substance. The authors have observed a different healing dynamic resulting from extensive mandibular bone reduction, whereby the inflammatory changes observed for the first several days may be related and proportional to the quantities of harvested bone removed. Changes in the healing pattern of the more extensive surgical cases involving removal or reduction of exostoses can occur that are actually within a normal range to the experienced clinician but might appear bizarre and of considerable concern to others. These changes include a floor of the mouth lingual hematoma with diffuse edematous enlargements and color changes.

A hematoma is defined as the collection of blood within soft tissues from hemorrhage leading to tissue enlargement. Excessively traumatized capillary beds extravasate blood into the perivascular connective tissue. Poor circulation at the site allows pooling of blood, prostaglandin release, and a more profound inflammatory reaction. Hematoma formation to the face or body is a common result of trauma, an example of which is the "Black Eye." The floor of the mouth hematoma can be more problematic because of the fascial planes involved and the anatomic structures at this site.

Hematoma formation can usually be prevented or controlled at the time of surgery by judicious handling of tissues and the management of operative and postoperative bleeding. During flap reflection, intact elevation of the periosteum using a full thickness flap design includes the blood vessels within the flap. The soft tissues and the contained vascular bed reduce the surgical trauma more commonly experienced with periosteal perforation. ${ }^{2}$ Postoperatively, direct pressure on the surgical site helps insure close adaptation of the mucoperiosteum to bone, thereby, reducing the incidence of hematoma formation. ${ }^{3}$ However, the flap design and method of closure, using a trauma-free procedure, is the most important element in prevention.
Lingual hematomas have been documented as a result of maxillofacial trauma causing tongue laceration, ${ }^{4-7}$ post extraction complication, ${ }^{2,8-9}$ severe hypertension, ${ }^{10}$ unusual complications of anticoagulant therapy, ${ }^{11-13}$ and surgical implant placement. ${ }^{14-18}$ Although not recorded in the dental literature, periodontal flap procedures may also effect hematoma formation. While a relatively uncommon sequel of surgical therapy, the broad spectrum of events resulting in hematoma necessitate the dental practitioner's familiarity with its diagnosis and treatment.

The three case reports presented here describe a range of wound healing occurring in the first two to three days following mandibular torus reduction during periodontal surgery with the diagnosis, treatment, and outcomes discussed.

\section{Case Reports}

\section{Case 1}

A 41-year-old Caucasian female was referred for treatment of severe chronic periodontitis. Initial therapy consisted of home care instruction, scaling and root planning, followed by reevaluation. The lower left quadrant was then treated surgically. One carpule of $2 \%$ lidocaine with 1:100,000 epinephrine was administered to provide block anesthesia of the inferior alveolar nerve. This was followed by the administration of infiltration anesthesia around the surgical site using one carpule of $2 \%$ lidocaine with $1: 50,000$ epinephrine. Sulcular incisions buccally and lingually allowed the elevation of full thickness mucoperiosteal flaps in an envelope fashion exposing contaminated roots and supporting periodontium. The exposed roots were scaled and root planed, followed by osseous recontouring around teeth \#17-24. Residual infrabony defects around teeth \#17 and \#18 were managed by the placement of autogenous bone, grafted from a torus reduction in the premolar region. The torus was reduced using a surgical handpiece and \#6 surgical bur to produce a mash coagulum. This coagulum graft material was used to fill each bony defect. The flaps were coapted for primary intention healing with one 4-0 continuous and four interrupted 4-0 silk sutures. A Coe-Pak ${ }^{\circledR}$ (GC America Inc., Alsip, II, USA) was placed. The patient was dismissed without surgical complication. Prescriptions for 28 tablets of Motrin 
$600 \mathrm{mg}$ to be taken $1 \mathrm{tab} \mathrm{q} 6 \mathrm{~h}$ and for 11 tablets of Doxycycline $100 \mathrm{mg}$ to be taken 2 tabs stat, then one tab daily were provided for the patient. In addition, chlorhexidine gluconate mouthrinse (Peridex ${ }^{\circledR}$, AstraZeneca Pharmaceuticals LP, Wilmington, DE, USA) was prescribed twice a day for 30 seconds to commence 24 hours after surgery.

Alarmed by the appearance of the floor of her mouth, the patient called and was examined the next morning. The clinical examination revealed a large hematoma that crossed the midline of the floor of the mouth (Figure 1).

The patient had no elevated temperature, free movement of her tongue, and no difficulty breathing or swallowing. Doxycycline was discontinued and 21 tablets of $500 \mathrm{mg}$ amoxicillin to be taken one tablet three times daily was prescribed. The chlorhexidine gluconate (Peridex) prescription was continued.

This new antibiotic regime was instituted empirically to reduce the potential for a floor of the mouth fascial plane infection. Fortunately, the patient did not take doxycycline as prescribed initially so there was no biologic interference with the bactericidal activity of the amoxicillin. Follow up instructions included home monitoring of her temperature, glandular areas, and changes in tongue or swallowing movements. The patient was called for a follow-up on her status eight hours later and again 24 hours later. Healing progressed without intensified symptoms, and the sutures and dressing were removed after seven days. The improved tissue response at day seven is shown in Figure 2. After four weeks, clinical healing appeared complete.

\section{Case 2}

A 75-year-old Caucasian female, diagnosed with generalized moderate to severe chronic periodontitis, presented for periodontal surgery on the lower left quadrant (\#17-22) after nonsurgical initial preparation. The patient related an insignificant medical history, an allergy to sulfur, and a daily $80 \mathrm{mg}$ prophylactic aspirin regimen that was discontinued seven days prior to surgery.

One carpule of $2 \%$ lidocaine with 1:100,000 epinephrine was administered for an inferior

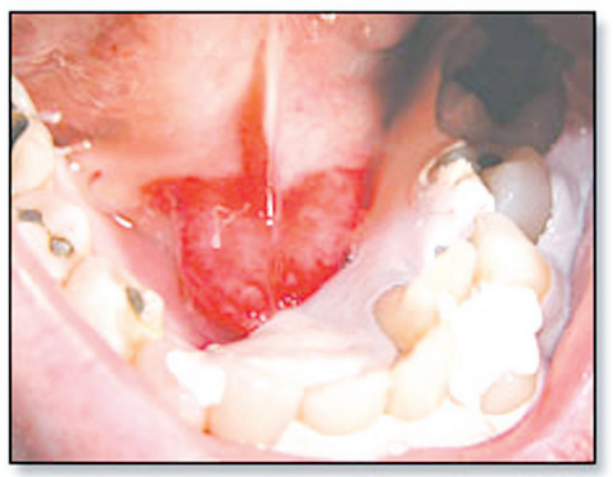

Figure 1. View of the hematoma on the morning after periodontal surgery on the mandibular left quadrant. Note the edema and erythema of the floor of the mouth.

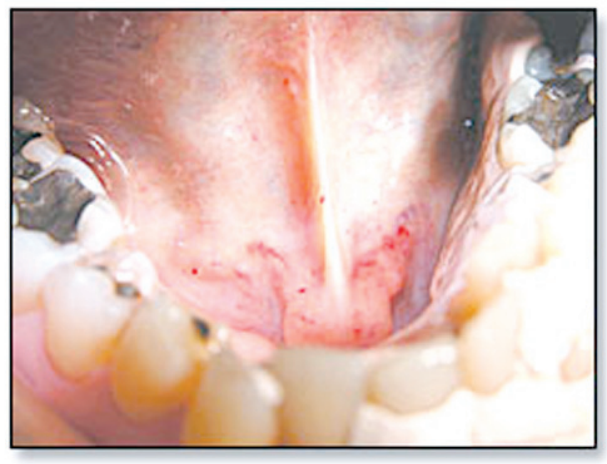

Figure 2. Seven days after periodontal surgery. Note the dramatic improvement.

alveolar nerve block followed by local infiltration around the surgical site with one carpule of $2 \%$ lidocaine with 1:50,000 epinephrine. Sulcular incisions on the buccal and lingual surfaces facilitated the release of full thickness mucoperiosteal flaps. After marginal bone recontouring, a residual two-wall infrabony defect was observed around teeth \#17 and \#18. In addition, a Class II buccal furcation lesion was probed on \#18. Bone grafting material was harvested from the mandibular lingual torus and other exostoses in the premolar and molar areas and combined with a bovine Bio-Oss ${ }^{\circledast}$ xenograft (Geistlich Pharma AG, Wolhusen, Switzerland) for placement within the defects. A porcine collagen membrane was used to cover the grafted site, and the flaps were re-approximated with five 4-0 PLA-PGA Vicryl sutures (Ehicon, mantzikosville, NJ, USA) to achieve primary closure. The collagen barrier extended approximately three millimeters beyond the grafted sites and was fully submerged below the flaps. A Coe Pak ${ }^{\circledast}$ surgical dressing was placed on the lingual surface only. 


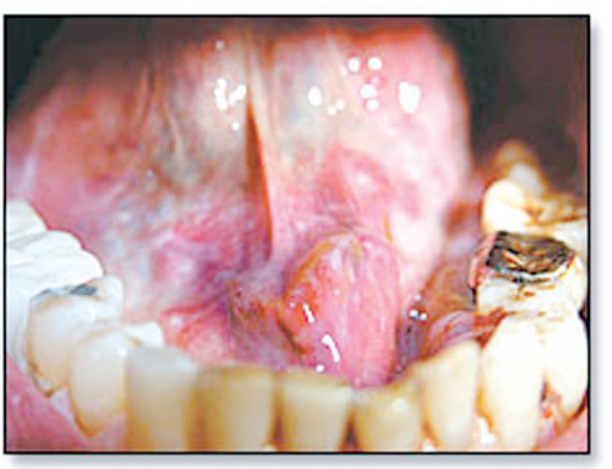

Figure 3. Morning after periodontal surgery on the mandibular left quadrant. The patient was still experiencing bleeding from the lingual free gingival margin.

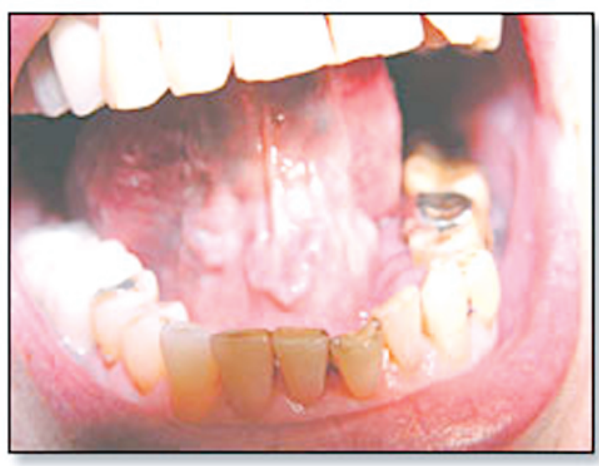

Figure 5. One week after initial surgery hematoma is almost completely dissipated.

The patient was dismissed without surgical complication; Motrin $600 \mathrm{mg}$ was dispensed for analgesia. Following our clinical protocol, 21 tablets of $500 \mathrm{mg}$ amoxicillin, taken as one tablet three times daily, were prescribed as a supplement to the guided tissue regeneration procedure. Chlorhexidine gluconate $0.12 \%$ was prescribed as a rinse twice a day for 30 seconds to commence 24 hours after surgery.

The patient was seen the following morning with concern about a gross soft tissue enlargement and discoloration beneath her tongue along with light bleeding. The clinical examination revealed diffuse inflammation and hematoma formation extending below the tongue involving the anterior floor of the mouth (Figures 3 and 4).

At no time did the patient suffer difficulty in breathing or swallowing. There was no elevation of temperature and no marked lymphadenopathy. The floor of the mouth was irrigated with cold

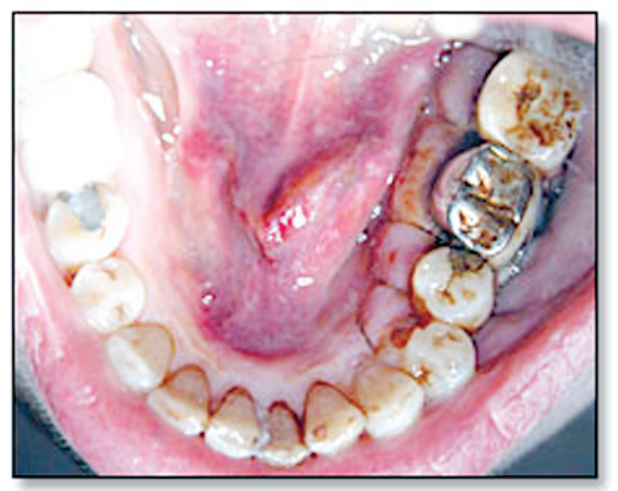

Figure 4. Hematoma crossing the midline and causing elevation of the floor of the mouth.

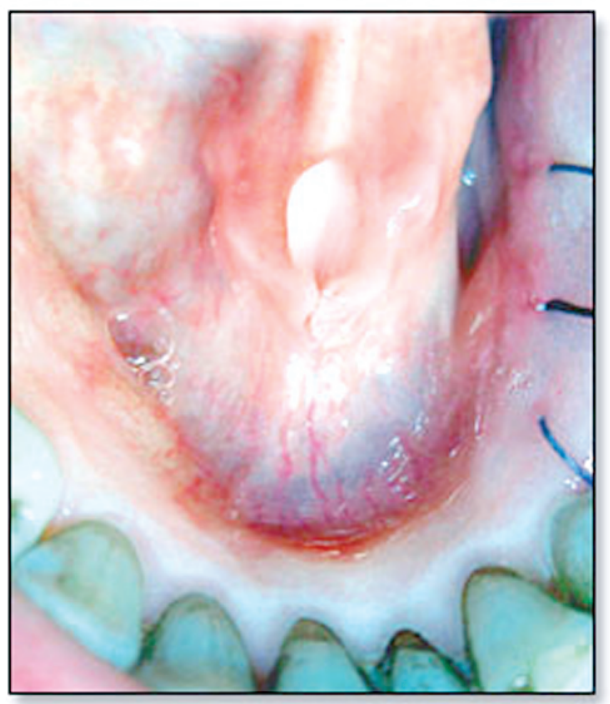

Figure 6. Floor of the mouth anatomy and salivary function is normal after only one week.

saline and pressure applied by replacing the original dressing. The patient was instructed to continue with the antibiotic as initially prescribed, and she was rescheduled for observation on day two and day seven (Figures 5 and 6). At these times, clinical observation demonstrated a resolving inflammation, reduced hematoma, and healing without incident.

\section{Case 3}

A 60-year-old African American male under treatment for moderate to advanced chronic periodontitis was treated surgically on the lower left quadrant (\#17-23). The medical history revealed a congenital heart murmur so the patient 


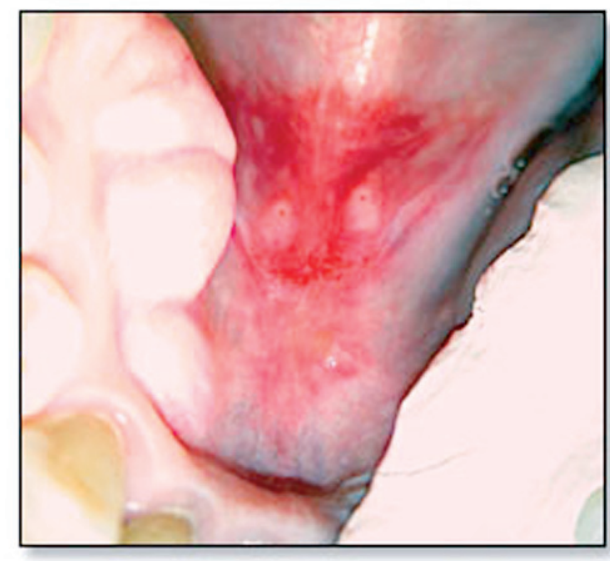

Figure 7. Ecchymosis one day after periodontal surgery.

was premedicated with 2 grams of amoxicillin one hour before the procedure as recommended by the American Heart Association and the American Dental Association. One carpule of $2 \%$ lidocaine with 1:100,000 epinephrine was administered for an inferior alveolar block and supplemented with infiltration local anesthesia using one carpule 2\% lidocaine with 1:50,000 epinephrine. Sulcular incisions allowed the elevation of full thickness mucoperiosteal flaps exposing teeth roots and marginal supporting bone. Scaling and root planing, ostectomy, and osteoplasty were performed to provide a more ideal supporting architecture. However, residual angular (vertical) defects were prepared around teeth 17, 18, 20 , and 21 to receive autogenous bone from the reduction of large lobulated lingual tori. The lobulated tori on the opposite lingual surface, which were bilaterally symmetrical to the tori, were reduced during this surgery as illustrated in Figure 7.

In a similar fashion to the other cases presented torus reduction was accomplished with a \#6 round surgical carbide bur in a surgical handpiece, collecting a bone coagulum for implantation. ${ }^{19}$ For this procedure, Collatape (Integra LifeSciences, Plainsboro, NJ, USA) was placed over the reduced bone surface beneath the flap to help promote platelet aggregation and more rapid clot formation. The flaps were coapted for primary closure with one 4-O silk continuous suture. Coe$\mathrm{Pak}^{\circledast}$ was placed and post operative instructions given. Twenty-eight tablets of $600 \mathrm{mg}$ Motrin were dispensed to be taken as one tablet every four to six hours. Twenty-eight tablets of Clindamyacin
$150 \mathrm{mg}$, to be taken four times daily, were prescribed for a seven day period. Clindamyacin was used instead of amoxicillin; this is in accordance with the American Heart Association's recommendation to use a different antibiotic than to prescribe the same one used for premedication of the patient. Listerine ${ }^{\boxplus}$ (Pfizer, Morris Plains, NJ, USA), an essential oil mouthrinse, was recommended.

To assess the role that increased pressure at the wound site may play at reducing hematoma, the patient was instructed to maintain a bolus of gauze placed in the sublingual cavity below and medial to the surgical dressing. The bolus of gauze was to be replaced every 30 minutes for the remainder of the day. The patient was recalled after 24 hours for observation. At that time, the sublingual area showed diffuse inflammation but far less tissue elevation, fluidity, and bulk (Figure 7). The patient was seen in seven days as a follow up to the surgery with the healing progressing uneventfully.

\section{Discussion}

The authors differentiate wound healing responses post-torus reduction on the lingual aspect of the mandible from other surgery in a number of ways. First, the initial response seen within 24 to 48 hours after extensive bone reduction is magnified and dramatic compared to the tissue response observed after procedures with less bone removal. A possible explanation is the relationship between quantity of cellular perturbation, the increase in prostaglandin E2 release, and the magnification of the process of inflammation..$^{20-21}$ The literature discusses this relationship when comparing arthroscopic wound healing to the more traditional surgical responses in vascular, ${ }^{22}$ orthopedic, ${ }^{23}$ gastrointestinal, ${ }^{24}$ and genito-urinary surgery. ${ }^{25-26}$ The more cell membranes perturbed, the greater the release of acute phase reactants resulting in a greater inflammatory response. ${ }^{27}$
Although hematoma formation is more pronounced than are other interstitial blood presentations, such as purpura or ecchymosis, most occurrences respond favorably with time and normal healing. The hematoma occurring as a result of extensive bone reduction on the lingual surface of the mandible is worrisome because 
of the anatomic site and the potential for serious infection through loose areolar fascial planes. Several publications detail potential infection ${ }^{9}$ or vascular injury ${ }^{4-7}$ leading to serious morbidity and potential mortality as a result of airway obstruction by immense enlargement of the sublingual hematoma. Sublingual hematomas have been shown to extend from lingual surgery as distant to the intraoral site as the mediastinal area. ${ }^{17}$ These reports demonstrate the potential of interstitial hemorrhage extending a substantial distance without infection.

The clinician can best determine post operative infection by assessing the presence of fever, lymphadenopathy, and malaise. These symptoms can develop from infection as a prelude to tongue movement and swallowing difficulties. The literature supports the impact these clinical findings have on diagnosis and patient management in the following manner:

Fever occurs through two biologic mechanisms, the central action of bacterial pyrogens and prostaglandin E2 on the hypothalamic temperature control center. ${ }^{19,28-29}$ Fever usually indicates bacterial contamination and/or abnormally high prostaglandin production by cell death or cell membrane perturbation. Lymphadenitis indicates excessive glandular output of B and T cells in response to infection. ${ }^{30}$ In floor of the mouth infections, lymphadenitis of the mental, submaxillary, and superficial cervical lymph nodes enlarge and are painful to palpation. ${ }^{31}$ Malaise by acute patient history or presentation is a subjective finding; however, experienced clinicians recognize malaise when it is produced. The increased biologic burden on the host versus bacterial challenge requires unusual energy expenditure and systemic weakness recognized as descriptive malaise.

Floor of the mouth hematomas, evaluated during the first 24 to 48 hours, without concomitant symptoms of infection or disturbance in breathing and swallowing are monitored without

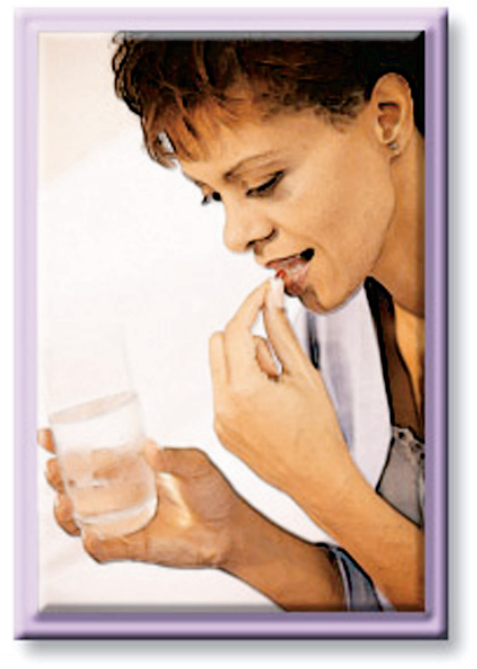

intervention. Soft tissue manipulation at this early stage of healing can be counter-productive, as additional quantities of inflammatory biochemicals slow the healing process. ${ }^{27}$

When the treatment plan is to reduce tori and other exostoses, the authors recommend the empirical administration of an antibiotic preoperatively to encourage bone graft success and post operatively to reduce the potential for floor of the mouth infection. A second defensible supplement to periodontal surgery would be a non-steroidal anti-inflammatory drug (NSAID) regimen reducing the impact of prostaglandin production from the surgery. Both Malamed ${ }^{32}$ and Jackson et al. ${ }^{33}$ recommend the administration of 800 mgs Ibuprofen one hour prior to surgery, followed by 800 mgs lbuprofen twice daily. Dionne et al. ${ }^{34}$ also recommend affirming the analgesic and anti-inflammatory effectiveness of this regimen, emphasizing the safety of this therapeutic dose at 1600 mgs per day within the maximal allowable dose of 3200 mgs per day.

Case reports ${ }^{9,15-16}$ in the dental literature suggest the application of pressure at the surgical site reduces hematoma formation. These case reports are impressions and not controlled studies. In addition, they reference other case reports employing pressure to reduce interstitial bleeding. Acknowledging a single case report influenced these authors, we modified our surgical management by instructing patient (Case \#3) to maintain gauze pressure on the floor of the mouth adjacent to the surgical site. This 
patient continued the pressure regiment for the remainder of the day, changing the gauze every 30 minutes. This regimen was different than the first two cases, where the patients were told to remove the gauze when they returned home. The clinical response was ecchymosis, interstitial bleeding without soft tissue elevations. The floor of the mouth hematoma characterized by surface enlargements did not develop. Although the immediate post operative surgical outcome was more favorable than observed in the first two case reports, the explanation is as anecdotal as the other published case reports, offering the readership little more than additional subjective impressions without the basis of controls.

Admittedly, at the 24 to 48 hour observations, accurately diagnosing floor of the mouth infection from non-infected hematomas is difficult, so prescribing an antibiotic regimen prophylactically seems a prudent recommendation. A recent meta-analysis of reported literature does show a slight statistical significant advantage offered by the administration of antibiotics prophylactically on periodontal outcomes. However, the difficulty in selecting the appropriate patient, antibiotic, and dosage precludes prescribing antibiotics for all procedures. $^{35}$

\section{Summary}

Floor of the mouth hematomas from periodontal surgery with torus and other exostoses reduction may be a common occurrence. These hematomas tend to resolve within a week and do not need further treatment. However, because of the severity of a non resolving hematoma, the patient should be made aware of the complication.

The patient should also be instructed to watch the floor of the mouth for any signs of swelling and tongue elevation and to call the dentist as soon as any breathing, swallowing, or speaking difficulties occur. Future studies should be initiated to observe the incidence of floor of the mouth hematomas. 


\section{References}

1. Kao R. Periodontal Regeneration and Reconstructive Surgery. In: Rose L, Mealey, B., Genco, R., Cohen D., editor. Periodontics, Medicine, Surgery and Implants. St. Louis: Mosby; 2004. p. 573.

2. Hunt PR. Safety aspects of mandibular lingual surgery. J Periodontol 1976;47(4):224-9..

3. Osbon DB. Postoperative complications following dentoalveolar surgery. Dent Clin North Am 1973;17(3):483-504.

4. Hing NR, Bowler MD, Byth PL, Daly CG. Lingual haematoma leading to upper airway obstruction. Br J Oral Maxillofac Surg 1985;23(5):322-5.

5. Ng KP. Lingual haematoma: yet another unusual cause of upper airway obstruction. Med J Malaysia 1998;53(1):112-4.

6. Chase CR, Hebert JC, Farnham JE. Post-traumatic upper airway obstruction secondary to a lingual artery hematoma. J Trauma 1987;27(8):953-4.

7. Kattan B, Snyder HS. Lingual artery hematoma resulting in upper airway obstruction. J Emerg Med $1991 ; 9(6): 421-4$.

8. Sakamoto E, Miller R, Stratigos GT, Arthur A. Serious postextraction hemorrhage into the submandibular space: report of case. J Am Dent Assoc 1975;90(3):654-8.

9. Goldstein $\mathrm{BH}$. Acute dissecting hematoma: a complication of oral and maxillofacial surgery. J Oral Surg 1981;39(1):40-3.

10. Prepageran N, Raman R, Ismail SM, Rahman ZA. Spontaneous sublingual hematoma as a complication of severe hypertension: first report of a case. Ear Nose Throat J 2002;81(8):576-8.

11. Murray JM, Blunnie WP. Acute upper airway obstruction following sub-lingual haematoma. Ir Med $\mathrm{J}$ 1983;76(11):458.

12. Riggs JE. Sublingual hematoma: an unusual complication of heparin anticoagulation. W V Med J 1986;82(5):131.

13. Kaynar AM, Bhavani-Shankar K, Mushlin PS. Lingual hematoma as a potential cause of upper airway obstruction. Anesth Analg 1999;89(6):1573-5.

14. Boyes-Varley JG, Lownie JF. Haematoma of the floor of the mouth following implant placement. Sadj 2002;57(2):64-5.

15. Niamtu J, 3rd. Near-fatal airway obstruction after routine implant placement. Oral Surg Oral Med Oral Pathol Oral Radiol Endod 2001;92(6):597-600.

16. Givol N, Chaushu G, Halamish-Shani T, Taicher S. Emergency tracheostomy following lifethreatening hemorrhage in the floor of the mouth during immediate implant placement in the mandibular canine region. J Periodontol 2000;71(12):1893-1895.

17. Mordenfeld A, Andersson L, Bergstrom B. Hemorrhage in the floor of the mouth during implant placement in the edentulous mandible: a case report. Int J Oral Maxillofac Implants 1997;12(4): 558-61.

18. Kalpidis CD, Setayesh RM. Hemorrhaging associated with endosseous implant placement in the anterior mandible: a review of the literature. J Periodontol 2004;75(5):631-45.

19. Robinson E. Osseous coagulum for bone induction. J Periodontol 1969;40(9):503-10.

20. Murakami M, Kudo I. Recent advances in molecular biology and physiology of the prostaglandin E2-biosynthetic pathway. Prog Lipid Res 2004;43(1):3-35.

21. Harris SG, Padilla J, Koumas L, Ray D, Phipps RP. Prostaglandins as modulators of immunity. Trends Immunol 2002;23(3):144-50.

22. Cerveira JJ, Halpern VJ, Faust G, Cohen JR. Minimal incision abdominal aortic aneurysm repair. J Vasc Surg 1999;30(6):977-84.

23. Jaikumar S, Kim DH, Kam AC. History of minimally invasive spine surgery. Neurosurgery 2002;51(5 Suppl):S1-14.

24. Guller U, Jain N, Hervey S, Purves H, Pietrobon R. Laparoscopic vs open colectomy: outcomes comparison based on large nationwide databases. Arch Surg 2003;138(11):1179-86.

25. Dumanian GA, Denham W. Comparison of repair techniques for major incisional hernias. Am J Surg 2003;185(1):61-5.

26. Gill IS. The case for laparoscopic adrenalectomy. J Urol 2001;166(2):429-36. 
27. Ebersole JL, Cappelli D. Acute-phase reactants in infections and inflammatory diseases. Periodontol 2000 2000;23:19-49.

28. Blatteis CM, Sehic E, Li S. Pyrogen sensing and signaling: old views and new concepts. Clin Infect Dis 2000;31 Suppl 5:S168-77.

29. Saper CB, Breder CD. The neurologic basis of fever. N Engl J Med 1994;330(26):1880-6.

30. Parham P. The Immune System. 2nd ed. New York: Garland Science. 2004. p. 17.

31. Dee R. Lymphatic Disorders. In: Beers M, Fletcher, A., Jones, T., Porter, R., editor. The Merck Manual of Medical Information. 2nd ed: Merck \& Co.; 2003. p. 239-42.

32. Malamed S. Handbook of Local Anesthesia. 5th ed. St. Louis: Mosby; 2004. p. 278.

33. Jackson DL, Moore PA, Hargreaves KM. Preoperative nonsteroidal anti-inflammatory medication for the prevention of postoperative dental pain. J Am Dent Assoc 1989;119(5):641-7.

34. Dionne RA, Campbell RA, Cooper SA, Hall DL, Buckingham B. Suppression of postoperative pain by preoperative administration of ibuprofen in comparison to placebo, acetaminophen, and acetaminophen plus codeine. J Clin Pharmacol 1983;23(1):37-43.

35. Haffajee AD, Socransky SS, Gunsolley JC. Systemic anti-infective periodontal therapy. A systematic review. Ann Periodontol 2003;8(1):115-81.

\section{About the Authors}

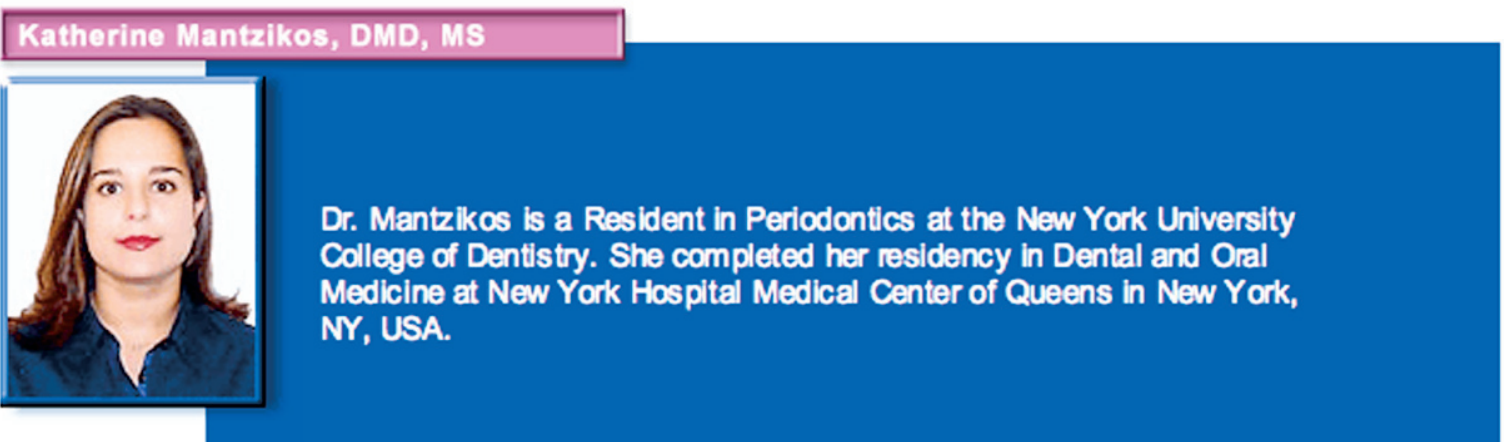

\section{Stuart L. Segelnick, DDS, MS}
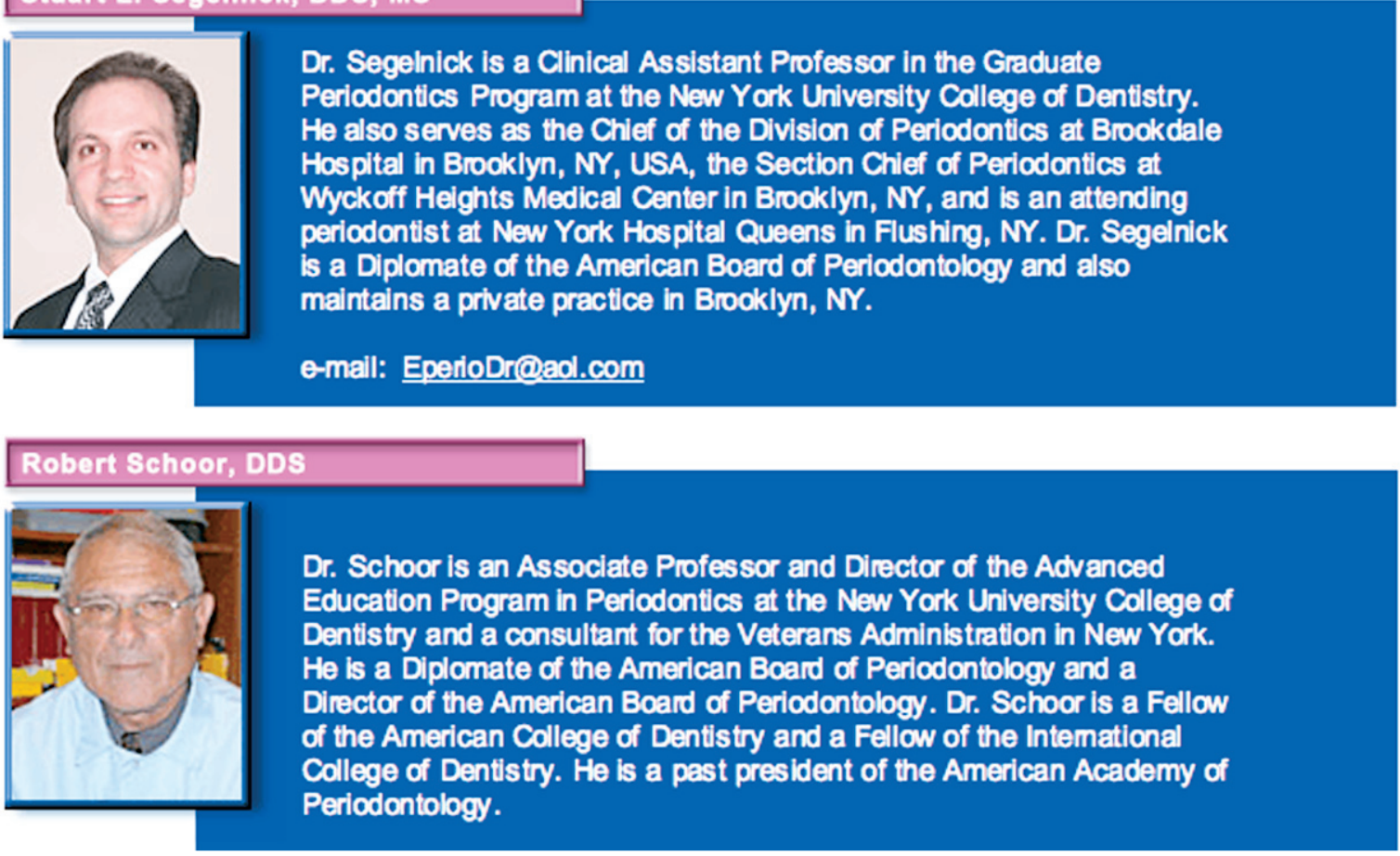